\title{
A!
}

\section{Aalto University}

This is an electronic reprint of the original article.

This reprint may differ from the original in pagination and typographic detail.

Sanchez, D.A.; Giebels, B.; Fortin, P.; Horan, D.; Szostek, A.; Fegan, S.; Baczko, A.-K.;

Finke, J.; Kadler, M.L.; Kovalev, Y.Y.; Lister, M.L.; Pushkarev, A.B.; Savolainen, T.

From radio to TeV: the surprising spectral energy distribution of AP Librae

Published in:

Monthly Notices of the Royal Astronomical Society

DOI:

$10.1093 /$ mnras/stv2151

Published: 01/01/2015

Document Version

Publisher's PDF, also known as Version of record

Please cite the original version:

Sanchez, D. A., Giebels, B., Fortin, P., Horan, D., Szostek, A., Fegan, S., Baczko, A-K., Finke, J., Kadler, M. L., Kovalev, Y. Y., Lister, M. L., Pushkarev, A. B., \& Savolainen, T. (2015). From radio to TeV: the surprising spectral energy distribution of AP Librae. Monthly Notices of the Royal Astronomical Society, 454(3), 3229-3239. https://doi.org/10.1093/mnras/stv2151

This material is protected by copyright and other intellectual property rights, and duplication or sale of all or part of any of the repository collections is not permitted, except that material may be duplicated by you for your research use or educational purposes in electronic or print form. You must obtain permission for any other use. Electronic or print copies may not be offered, whether for sale or otherwise to anyone who is not an authorised user. 


\title{
From radio to TeV: the surprising spectral energy distribution of AP Librae
}

\author{
D. A. Sanchez, ${ }^{1 \star}$ B. Giebels,${ }^{2}$ P. Fortin,${ }^{3}$ D. Horan, ${ }^{2}$ A. Szostek, ${ }^{4}$ S. Fegan, ${ }^{2}$ \\ A.-K. Baczko, ${ }^{5,6}$ J. Finke, ${ }^{7}$ M. L. Kadler ${ }^{6}$ Y. Y. Kovalev, ${ }^{8,9}$ M. L. Lister, ${ }^{10}$ \\ A. B. Pushkarev ${ }^{9,11,12}$ and T. Savolainen ${ }^{9,13}$ \\ ${ }^{1}$ Laboratoire d'Annecy-le-Vieux de Physique des Particules, Université de Savoie, CNRS/IN2P3, F-74941 Annecy-le-Vieux, France \\ ${ }^{2}$ Laboratoire Leprince-Ringuet, Ecole Polytechnique, CNRS/IN2P3, F-91128 Palaiseau, France \\ ${ }^{3}$ Fred Lawrence Whipple Observatory, Harvard-Smithsonian Center for Astrophysics, Amado, AZ 85645, USA \\ ${ }^{4}$ Kavli Institute for Particle Astrophysics and Cosmology, Department of Physics and SLAC National Accelerator Laboratory, Stanford University, Stanford, \\ CA 94305, USA \\ ${ }^{5}$ Dr Karl Remeis-Observatory ECAP, Astronomical Institute, Friedrich-Alexander University Erlangen-Nuremberg, Sternwartstr. 7, D-96049 Bamberg, \\ Germany \\ ${ }^{6}$ Lehrstuhl für Astronomie, Universität Würzburg, Campus Hubland Nord, Emil-Fischer-Strasse 31, D-97074, Würzburg, Germany \\ ${ }^{7}$ U.S. Naval Research Laboratory, Code 7653, 4555 Overlook Ave. SW, Washington, DC, 20375-5352, USA \\ ${ }^{8}$ Astro Space Center of Lebedev Physical Institute, Profsoyuznaya 84/32, 117997 Moscow, Russia \\ ${ }^{9}$ Max-Planck-Institut für Radioastronomie, Auf dem Hügel 69, D-53121 Bonn, Germany \\ ${ }^{10}$ Department of Physics, Purdue University, West Lafayette, IN 47906, USA \\ ${ }^{11}$ Crimean Astrophysical Observatory, 98409 Nauchny, Crimea, Russia \\ ${ }^{12}$ Pulkovo Observatory, Pulkovskoe Chaussee 65/1, 196140 St. Petersburg, Russia \\ ${ }^{13}$ Aalto University Metsähovi Radio Observatory, Metsähovintie 114, FI-02540 Kylmälä, Finland
}

Accepted 2015 September 14. Received 2015 September 1; in original form 2015 June 2

\begin{abstract}
Following the discovery of high-energy (HE; $E>10 \mathrm{MeV}$ ) and very-high-energy (VHE; $E>$ $100 \mathrm{GeV}) \gamma$-ray emission from the low-frequency-peaked BL Lac (LBL) object AP Librae, its electromagnetic spectrum is studied over 60 octaves in energy. Contemporaneous data in radio, optical and UV together with the (non-simultaneous) $\gamma$-ray data are used to construct the most precise spectral energy distribution of this source. The data have been found to be modelled with difficulties with single-zone homogeneous leptonic synchrotron self-Compton (SSC) radiative scenarios due to the unprecedented width of the HE component when compared to the lower-energy component. The two other LBL objects also detected at VHE appear to have similar modelling difficulties. Nevertheless, VHE $\gamma$-rays produced in the extended jet could account for the VHE flux observed by HESS.
\end{abstract}

Key words: galaxies: active-BL Lacertae objects: individual: AP Librae-galaxies: jets.

\section{INTRODUCTION}

Blazars are among the most energetic objects in the Universe that exhibit non-thermal electromagnetic spectra from radio up to veryhigh-energy (VHE; $E>100 \mathrm{GeV}$ ) $\gamma$-rays, with a two-component spectral energy distribution (SED) structure in a $v f(v)$ representation. Multi-wavelength data are of paramount importance to understand the mechanisms at play in the jet.

Blazars are divided into two classes: flat spectrum radio quasars (FSRQs) and BL Lacertae (BL Lac) objects, the latter being sub-divided into high-frequency-peaked BL Lac (HBL) and lowfrequency-peaked BL Lac (LBL). The distinction between HBL and
LBL classes is based on the low-energy peak position (Padovani \& Giommi 1995). HBL objects present a peak in the UV or X-ray range while the peak of LBL objects is located at lower energies (i.e. in optical wavelengths).

So far, the vast majority of BL Lac objects detected in VHE belong to the HBL sub-class. ${ }^{1}$ The SEDs of HBL objects are often successfully modelled with a synchrotron self-Compton (SSC) model, in which the low-energy emission is produced by synchrotron radiation of relativistic electrons, and the high-energy (HE) component by inverse Compton-scattering off the same synchrotron photons. HBL are the dominant class of extragalactic objects detected by

\footnotetext{
${ }^{1}$ To keep track of the number of detected object, an up-to-date VHE $\gamma$-ray catalogue can be found in the TeVCat http://tevcat.uchicago.edu
} 
ground-based Atmospheric Čerenkov Telescopes (ACTs) in the TeV $\gamma$-ray regime.

Only a few $\mathrm{TeV}$ emitters belong to the LBL sub-class and, among them, AP Librae ( $z=0.049$; Jones et al. 2009) was recently detected by the HESS collaboration (Abramowski et al. 2015) with a flux of $8.78 \pm 1.54_{\text {stat }} \pm 1.76_{\text {sys }} \times 10^{-12} \mathrm{~cm}^{-2} \mathrm{~s}^{-1}$ above $130 \mathrm{GeV}$ and a photon index $\Gamma=2.65 \pm 0.19_{\text {stat }} \pm 0.20_{\text {sys }}$ matching well the spectrum measured by the Fermi Large Area telescope (LAT) in the HE $(100 \mathrm{MeV}<E<300 \mathrm{GeV})$ range. Remarkably, the spectral break between the HE and VHE ranges is the smallest ever measured for an LBL object but cannot be explained by extragalactic background light (EBL) attenuation only (Sanchez, Fegan \& Giebels 2013). In this work, VHE and HE data have been extracted from Abramowski et al. (2015).

After the announcement of this detection by the HESS collaboration (Hofmann 2010), Swift and RXTE data were taken creating contemporaneous spectra in X-ray and $U V$ bands. Analysis and results are presented in Sections 2.1 and 2.3. Archival observation by Chandra (Section 2.2) has been analysed in this work, revealing the first X-ray extended jet for a VHE blazar. At longer wavelengths, AP Librae is one of the targets of different monitoring programs such as SMARTS (Section 2.3) and the MOJAVE program (Section 2.4), which provide long-term optical and VLBA measurements. The VHE detection, together with lower energy-data presented in this paper, enables us to draw the first complete SED of this source and to probe mechanisms at play in LBL objects. The broad-band SED is then discussed in the framework of different emission models in Section 3.

Throughout this paper a $\Lambda$ cold dark matter $(\Lambda \mathrm{CDM})$ cosmology with $H_{0}=71 \mathrm{~km} \mathrm{~s}^{-1} \mathrm{Mpc}^{-1}, \Omega_{\Lambda}=0.73$ and $\Omega_{\mathrm{M}}=0.27$ is assumed, resulting in a luminosity distance for AP Librae of $D_{\mathrm{L}}=215 \mathrm{Mpc}$ and a linear scale of $0.947 \mathrm{kpc}$ per arcsec (Wright 2006).

\section{MULTI-WAVELENGTH OBSERVATIONS}

\subsection{Swift-XRT and RXTE-PCA observations}

X-ray observations of AP Librae during the period of interest were retrieved using the HEASARC archive. Four consecutive daily observations (Obs ID 95141) of $\simeq 3 \mathrm{ks}$ each were carried out between 2010 July 10 and 14 with RXTE (Jahoda et al. 1996), with a total exposure of $\simeq 13 \mathrm{ks}$. The STANDARD2 RXTE-Proportional Counter Array (PCA) data were extracted using the FTOOLS in the HEASOFT 6.16 software package provided by NASA/GSFC and filtered using the RXTE Guest Observer Facility recommended criteria. Only signals from the top layer (X1L and X1R) of Proportional Counter Unit 2 (PCU2) were used to extract spectra in the $3-50 \mathrm{keV}$ range, using the faint-background model. The obtained daily light curve has an average rate of 0.44 counts s$^{-1}$, a variance of 0.03 counts s $\mathrm{s}^{-2}$ compatible with its expected variance of 0.02 counts s$^{-2}$ if the source were constant, and a chi-square probability of constancy of 27 per cent, hence no variability is present over the span of $4 \mathrm{~d}$.

During the period of interest, seven observations were carried out by the Swift mission (Burrows et al. 2005), between 2010 February 20 and 2011 August 16 (Obs ID 36341005 to 36341011), of which one 5 ks observation was carried out on 2010 July 7, near the RXTE observation. However, the short observation in Obs ID 36341009 was skipped. The photon-counting (PC) mode data are processed with the standard XRTPIPELINE tool (HEASOFT 6.16), with the source and background-extraction regions defined as a 20-pixel (4.7 arcsec) and a 40-pixel radius circle, respectively, the latter being centred nearby the former without overlapping. All exposures show a source with a stable average count rate of $\simeq 0.12$ counts $\mathrm{s}^{-1}$. Also the large $5 \mathrm{ks}$ XRT-PC light curve shows the source with an average count rate of $(0.13 \pm 0.02) \mathrm{s}^{-1}$ and an rms of $\simeq 0.01 \mathrm{~s}^{-1}$ for which no variability could be found with a 99 per cent confidence level upper limit on the fractional variance (as defined in Vaughan et al. 2003) $F_{\text {var }}$ of 0.95 . Using this count rate in WebPIMMS from HEASARC, an $R X T E$-PCA count rate of $\simeq 0.6$ counts $\mathrm{s}^{-1}$ is predicted, compatible with the value actually observed of 0.44 counts $\mathrm{s}^{-1}$ hinting at the fact that the source was probably in the same state during observations of both observatories. Given the low count rate, no pile-up is expected in PC mode, which is confirmed by the acceptable fit of a King profile to the PSF of all observations.

Spectral fitting of all Obs IDs was performed with PYXSPEC v1.0.4 (Arnaud 1996), using a response matrix for the combined PCA data set generated by the FTOOL PCARSP v11.7.1, and dedicated Ancillary Response Functions (ARFs) for all XRT data sets generated by XRTMKARF (along with the latest spectral redistribution matrices SWXPC0TO12s6_20110101v014 from CALDB). Spectra from all Obs IDs were rebinned to have at least 20 counts per bin using GRPPHA, channels 0 to 29 were ignored in the XRT-PC data, and only the $3-50 \mathrm{keV}$ range is used in the PCA data. All data sets are fitted to a power-law model $\mathrm{d} N / \mathrm{d} E=N_{0}\left(E / E_{0}\right)^{-\Gamma_{\mathrm{X}}}$, where $N_{0}$ is the normalization factor at a chosen reference energy $E_{0}=1 \mathrm{keV}$ and $\Gamma_{\mathrm{X}}$ the photon index. Using the Leiden/Argentine/Bonn (LAB) Survey of Galactic H I (Kalberla et al. 2005) weighted average hydrogen column density of $N_{\mathrm{H}}=8.14 \times 10^{20} \mathrm{~cm}^{-2}$, good fits are obtained for the power-law function $\left[P\left(\chi^{2}\right)=0.18-0.9\right]$ with a photon index of $\Gamma_{\mathrm{X}} \simeq 1.55$ on average. All XRT observations were also summed, a new exposure file built with XIMAGE, and a new ARF for the summed spectrum. This latter spectrum extends up to $\simeq 7 \mathrm{keV}$. Another spectrum was derived this time limited to 1 count/bin to allow an extension to higher energies, and was fitted using STATISTIC CSTAT required in the case of Poisson data. The fit parameters are entirely compatible with those obtained using $\chi^{2}$ statistics, but the spectrum extends up to $\simeq 10 \mathrm{keV}$. All fit parameters, along with the unabsorbed $0.3-10 \mathrm{keV}$ flux $F_{0.3-10 \mathrm{keV}}$ (retrieved for each flux using CFLUX), are shown in Table 1 and the light curve is shown in Fig. 1.

Systematic errors on the Swift-XRT spectra and absolute flux are less than 3 per cent and 10 per cent, respectively (Godet et al. 2009), while PCA-XRT cross-calibration details can be found in Tsujimoto et al. (2011).

\subsection{Chandra observations}

AP Librae was observed by Chandra on 2003 July 4 with a total exposure time of $14 \mathrm{ks}$. The Chandra data reprocessing and reduction were performed following the standard procedures described in the Chandra Interactive Analysis of Observations ${ }^{2}$ (CIAO) threads, using CIAO v4.3 and the Chandra Calibration Database (CALDB) version 4.4.6. The data reveal the presence of an extended jet on arcsec scales, which is unique amongst the VHE emitting BL Lac class so far. A radio VLA observation was used to align the nuclear X-ray emission with the radio core. A registered, exposurecorrected and adaptively smoothed image of AP Librae in units of $\mathrm{ph} \mathrm{cm}^{-2} \mathrm{~s}^{-1} \mathrm{px}^{-1}$, with radio contours overlaid, is shown in Fig. 2. In order to assess to what degree the RXTE and Swift spectra need

\footnotetext{
${ }^{2}$ http://cxc.harvard.edu/ciao/index.html
} 
Table 1. Results of the spectral fitting of all XRT-PC and PCA observations.

\begin{tabular}{lccccc}
\hline Obs ID & $\begin{array}{c}\text { Time } \\
\text { MJD-5500 }\end{array}$ & $\begin{array}{c}N_{0} \\
\mathrm{ph} \mathrm{cm}^{-2} \mathrm{~s}^{-1}\end{array}$ & $\Gamma_{X}$ & $\begin{array}{c}P\left(\chi^{2}\right) \\
\text { percent }\end{array}$ & $\begin{array}{c}F_{0.3-10 \mathrm{keV}} \\
\times 10^{-12} \mathrm{erg} \mathrm{cm}^{-2} \mathrm{~s}^{-1}\end{array}$ \\
\hline 00036341005 & $247.2-247.2$ & $(9 \pm 1) \times 10^{-4}$ & $1.62 \pm 0.14$ & 18 & $6.8 \pm 0.8$ \\
00036341006 & $249.5-249.7$ & $(9 \pm 1) \times 10^{-4}$ & $1.45 \pm 0.09$ & 70 & $7.6 \pm 0.6$ \\
00036341007 & $384.7-384.8$ & $(8.3 \pm 0.4) \times 10^{-4}$ & $1.47 \pm 0.06$ & 31 & $7.2 \pm 0.4$ \\
00036341008 & $608.1-608.2$ & $(10 \pm 1) \times 10^{-4}$ & $1.49_{-0.14}^{+0.15}$ & 35 & $8.3_{-0.9}^{+1.0}$ \\
00036341010 & $608.0-608.0$ & & $1.51 \pm 0.09$ & 94 & $7.8 \pm 0.6$ \\
00036341011 & $609.8-609.9$ & $(9.3 \pm 0.5) \times 10^{-4}$ & $1.52_{-0.06}^{+0.07}$ & 60 & $7.6 \pm 0.4$ \\
Sum all above & & $(9.2 \pm 0.2) \times 10^{-4}$ & $1.52 \pm 0.02$ & 99 & $7.54 \pm 0.2$ \\
95141 & $387.9-391.9$ & $1.3_{-0.3}^{+0.4} \times 10^{-3}$ & $1.74 \pm 0.16$ & 91 & $5.6 \pm 0.4$ \\
\hline
\end{tabular}

corrections for non-core emission, the spectrum of the jet is estimated, with the caveat that this observation is not contemporaneous with the data set presented here.

A spectrum of the jet was taken from a polygon-shaped region which avoids the emission of the core and the ACIS readout streak. A core spectrum comes from a 2 arcsec region centred on the core. A background spectrum was extracted from four circular regions placed to the north and south of the source. The jet and background regions are marked in Fig. 2. In order to estimate the effects of pile-up in the core and jet region, the method described by Harris et al. (2011) was used. In the jet region no pile-up was found while it was necessary to correct for mild pile-up in the core.

The spectra of the core and the jet contain $\simeq 4900$ and $\simeq 200$ background-subtracted counts, respectively. Both spectra were binned to a minimum of 20 counts per bin, and fit in the $0.5-$ $7.0 \mathrm{keV}$ energy band using an absorbed power-law model in XSPEC with the same $N_{\mathrm{H}}$ as in Section 2.1. The fit of the jet spectrum yields a photon index $\Gamma_{\text {jet }}=1.59 \pm 0.16$ and a $2-10 \mathrm{keV}$ unabsorbed flux of $F_{2-10 \mathrm{keV}}^{\mathrm{jet}}=(1.07 \pm 0.37) \times 10^{-13} \mathrm{erg} \mathrm{cm}^{-2} \mathrm{~s}^{-1}$, with a $\chi^{2}=4.4$ for 7 dof, or more than an order of magnitude below the value measured for the source in Section 2.1 based on the Swift and RXTE data, which can hence safely be used as the X-ray flux of the core in AP Librae. The jet spectrum is comparable with the spectra of largescale quasar jets observed by Chandra, which may also be sources of relatively intense $\gamma$-ray emission (see the discussion in Sambruna et al. 2004; Finke, Dermer \& Böttcher 2008). Such a scenario is not formally excluded here since an extrapolation of the jet spectrum could connect within the experimental errors with either the HE or VHE fluxes reported here. Assuming no pileup, the best power-law fit to the core spectrum yields a photon index of $\Gamma_{\text {core }}=1.51 \pm 0.03$ and a $2-10 \mathrm{keV}$ unabsorbed flux of $F_{2-10 \mathrm{keV}}^{\text {core }}=3.18_{-0.14}^{+0.19} \times 10^{-12} \mathrm{erg} \mathrm{cm}^{-2} \mathrm{~s}^{-1}$. Using the PILEUP model in XSPEC, a pile-up corrected spectrum appears however to be softer with $\Gamma_{\text {core }}=1.68_{-0.06}^{+0.03}$ and $F_{2-10 \mathrm{keV}}^{\text {core }} \simeq 2.31 \times 10^{-12} \mathrm{erg}$ $\mathrm{cm}^{-2} \mathrm{~s}^{-1}$, with a $\chi^{2}=158.4$ for 129 dof. The pile-up model of Davis (2001) was used in the fit of the core spectrum, and the value of the pile-up parameter $\alpha>0$ indicates that the fit is indeed affected by this. However, it was not possible to obtain an error estimate on $\alpha$, and hence we also do not have an error estimate on the unabsorbed and pile-up corrected flux. Due to pile-up effects, the fit results for the core should be treated with caution. This extended X-ray jet was first reported by Kaufmann, Wagner \& Tibolla (2013). Our results differ slightly, probably because we used different extraction and background regions, and Kaufmann et al. did not take into account the above-mentioned ACIS readout streak.

\subsection{Swift-UVOT and SMARTS observations}

All of the available archival data taken on AP Librae with the ultraviolet and optical telescope (UVOT) on the Swift satellite were analysed. This comprised 35 exposures taken between 2007 April and 2010 July, 13 of which occurred during the time frame with which this paper is concerned (see Fig. 1). After extracting the source counts from an aperture of 5.0 arcsec radius around AP Librae and the background counts from four neighbouring regions, each of the same size, the magnitudes were computed using the UVOTSOURCE tool with calibrations from Breeveld et al. (2011). These were converted to fluxes using the values from Poole et al. (2008) after correction for extinction following the procedure and $R_{\mathrm{V}}$ value of Roming et al. (2009). The values of $a$ and $b$ from Roming et al. (2009), computed following the procedure of Cardelli, Clayton \& Mathis (1989), were used. The $E(B-V)$ value from Schlafly \& Finkbeiner (2011), accessed through the NASA/IPAC Extragalactic Database, was used. Results are summarized in Table 2.

AP Librae was observed in context of the Yale Fermi/sMARTS project $^{3}$ (Bonning et al. 2012). Observations were performed in the $B, R, J$ and $K$ bands between 2011 February 27 (MJD 55619) and 2013 March 3 (MJD 56739) and are shown in Fig. 1. The number of observations and the mean magnitudes are given in Table 2 together with the corresponding fluxes. Magnitudes have been corrected for Galactic absorption using values from Schlafly \& Finkbeiner (2011) and converted in flux units using the Bessell zero-points (Bessell 1990).

The host galaxy of AP Librae is bright and therefore the contribution from starlight must be taken into account to estimate the non-thermal flux from the core in the near-infrared to $U V$ band. The dereddened near-infrared and optical measurements of AP Librae reported in fig. 1 of Falomo et al. (1993), where the total emission was modelled with a giant elliptical galaxy template and a superposed non-thermal power-law continuum, are given for illustration in the composite SED of Fig. 3. The synchrotron emission probably peaks in the optical- to near-IR range, since the spectral index for AP Librae in that range is $\alpha_{\text {IROP }}=0.95 \pm 0.10$. In Hyvönen et al. (2007), the fluxes in the $B$ and $U$ bands were calculated for the host galaxy and the core. The fractional contribution of the latter was $\simeq 42$ per cent in the $B$ band and $\simeq 69$ per cent in the $U$ band. At higher energies the emission from the core accounts for an even higher percentage. To take this result into account, the host galaxy template of Silva et al. (1998) has been used and with a normalization adjusted to fit the data.

\footnotetext{
${ }^{3}$ http://www.astro.yale.edu/smarts/glast/pubs.html
} 


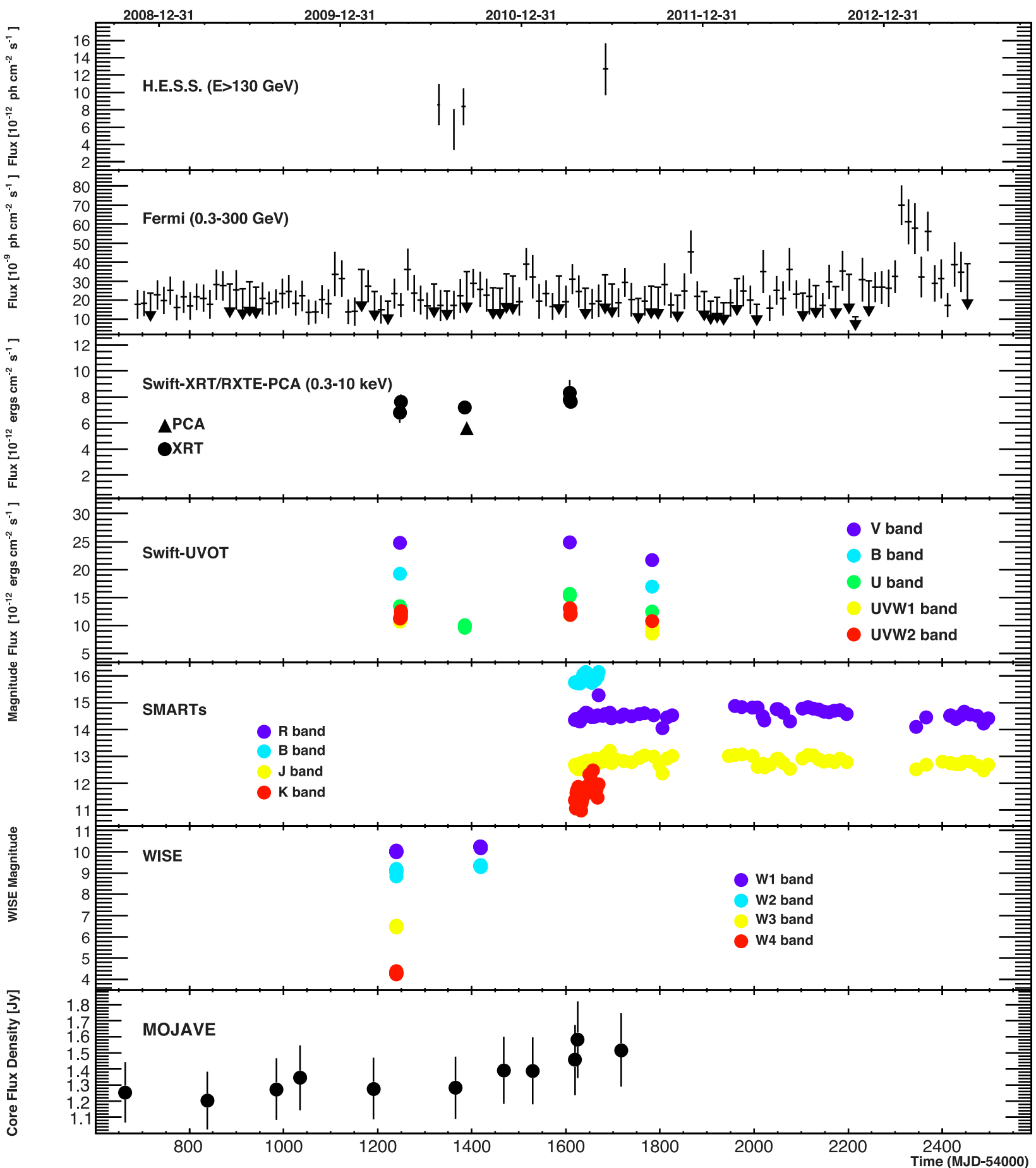

Figure 1. Light curves of AP Librae in, from top to bottom, VHE, HE, X-rays, UV, optical and radio (15 GHz) wavebands. The four RXTE observations (Obs ID 95141) were merged together and the seven Swift observations (Obs ID 36341005 to 36341011) are shown individually.

\subsection{MOJAVE}

The parsec-scale structure of the radio jet of AP Librae has been monitored throughout the past decade as part of the MOJAVE program ${ }^{4}$ (Monitoring of Jets in Active galactic nuclei with VLBA Experiments) with the Very Long Baseline Array (VLBA) at a frequency

\footnotetext{
${ }^{4}$ http://www.physics.purdue.edu/astro/MOJAVE
}

of $15 \mathrm{GHz}$. The VLBA data have been calibrated and analysed following the procedures described by Lister et al. (2009). The source shows a bright, continuous inner jet region with a bright jet core, i.e. apparent jet base, extending towards the South. At a resolution of typically $\simeq(1.5 \times 0.5)$ milli-arcsecond (mas), the core is not clearly separated from the inner jet. Elliptical Gaussian components were used to model the brightness distribution and to determine radio flux densities of different emission regions within the source. For the comparison with higher-energy multiwavelength data, we 


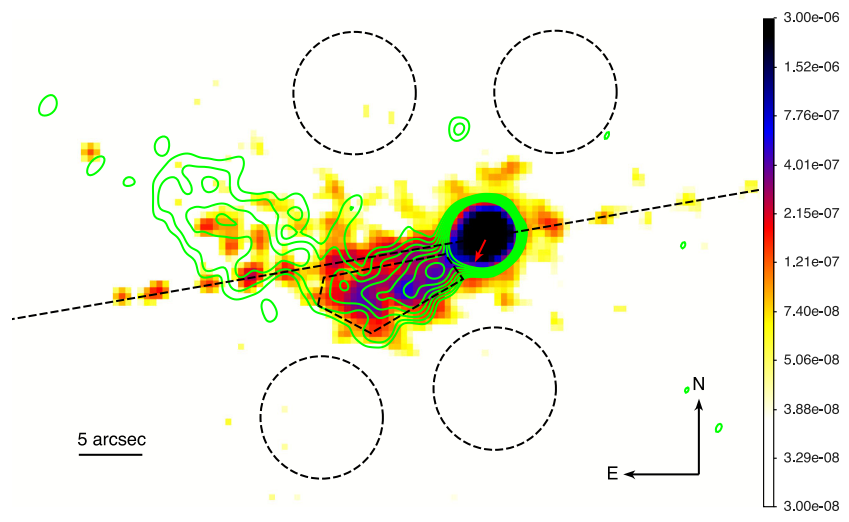

Figure 2. Adaptively smoothed, exposure-corrected X-ray image obtained by Chandra in the $0.5-7 \mathrm{keV}$ energy band in units of $\mathrm{ph} \mathrm{cm}^{-2} \mathrm{~s}^{-1} \mathrm{px}^{-1}$ with the pixel size of $0.492 \mathrm{arcsec}$, where 1 arcsec corresponds to $0.947 \mathrm{kpc}$ on linear scale. Overlaid are $1.4 \mathrm{GHz}$ radio emission contours at 2 arcsec resolution from reprocessing archival VLA data (program AB700; C.C. Cheung, private communication). The flux densities of radio contours increase by a factor of 1.5 , starting from a value of five times the rms noise equal to $1.82 \times$ $10^{-4} \mathrm{Jy} \mathrm{beam}^{-1}$. The small red arrow in the radio core shows the orientation of the milliarcsecond scale radio jet seen in VLBA (see e.g. Lister, Marscher \& Gear 1998; Zensus et al. 2002, and references therein for a discussion on the radio jet at different scales). A black dashed polygon delimits the region used to calculate the jet spectrum, the dashed circles are the background regions and a black dashed line indicates the location of an ACIS readout streak.

Table 2. Summary of the Swift-UVOT and SMARTS results. Columns 1 and 3 give the filter and corresponding energies and the second column gives the number of observations. Magnitudes (Column 4) are not corrected for Galactic absorption. The last column gives the corrected flux.

\begin{tabular}{lcccc}
\hline Filter & $\begin{array}{c}N_{\text {Obs }} \\
\text { Energy } \\
(\mathrm{eV})\end{array}$ & $\begin{array}{c}\text { Magnitude } \\
\left(10^{-11} \mathrm{erg} \mathrm{cm}^{-2} \mathrm{~s}^{-1}\right)\end{array}$ \\
\hline SMARTS: & & & & \\
$J$ & 25 & 0.56 & $11.63 \pm 0.37$ & $1.96 \pm 0.78$ \\
$R$ & 79 & 0.99 & $12.76 \pm 0.17$ & $3.26 \pm 0.54$ \\
$B$ & 74 & 1.77 & $14.53 \pm 0.18$ & $1.88 \pm 0.34$ \\
Swift-UVOT: & & & & $1.35 \pm 0.18$ \\
$V$ & 3 & 2.30 & $15.18 \pm 0.04$ & \\
$B$ & 3 & 2.86 & $15.94 \pm 0.04$ & $1.69 \pm 0.06$ \\
$U$ & 7 & 3.54 & $15.68 \pm 0.04$ & $0.96 \pm 0.03$ \\
UVW1 & 6 & 4.72 & $15.88 \pm 0.05$ & $0.63 \pm 0.03$ \\
UVW2 & 10 & 6.12 & $16.12 \pm 0.05$ & $0.57 \pm 0.02$ \\
UVM2 & 3 & 5.57 & $16.09 \pm 0.06$ & $0.55 \pm 0.03$ \\
\hline
\end{tabular}

focused on the inner 1.5 mas $(\simeq 1.41 \mathrm{pc})$ region, which could typically be modelled with 2-3 Gaussian model components. We have used different models with circular and elliptical model components and tested the formal statistical model-fitting uncertainties of the total flux density, which turn out to be much smaller $[\lesssim(1-3)$ per cent $]$ than the absolute calibration uncertainty, which can be conservatively estimated to be of the order of $\lesssim 10$ per cent.

The 16 MOJAVE observations from MJD 53853 to 55718 do not show signs of significant variability in the VLBI core region. Fig. 3 shows the value of $1.48 \mathrm{Jy}$ of the radio flux density, averaged over the full observations, from the inner 1.5 mas jet core.

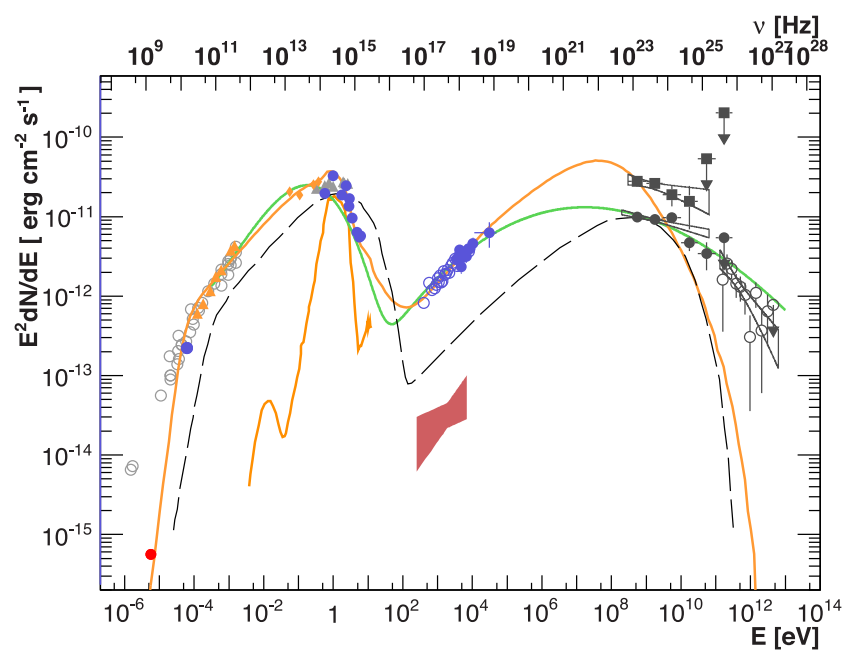

Figure 3. The broad-band SED of the LBL AP Librae. The orange triangles come from the Planck ERCSC. The orange diamonds are WISE measurements. Blue points are, from low energy to HE, MOJAVE $(15 \mathrm{GHz})$, Swift-UVOT (2.30-5.57 eV), SMARTS (0.56-2.86 eV), Swift-XRT/RXTE (3$50 \mathrm{keV}$ ). Grey points and butterflies are Fermi-LAT for the quiet (circle) and flare (square) periods $(0.3-300 \mathrm{GeV})$ and $\operatorname{HESS}(E>100 \mathrm{GeV})$ measurement from Abramowski et al. (2015). Swift-UVOT, sMARTs data are corrected for Galactic extinction and X-ray data are corrected for $N_{\mathrm{H}}$ absorption. Light grey data are taken from NED. The dark grey triangles come from Falomo et al. (1993). The red point is the radio flux of the extended jet. The orange line is the host galaxy template of Silva et al. (1998). The fit with two thirddegree polynomial functions, not corrected for EBL, are shown with a green line (see Section 3.1). The red butterfly is the Chandra spectrum from the jet. The dashed line is the SSC model from Tavecchio et al. (2010) whereas the red line is the model obtained in this work (see Table 4).

\section{DISCUSSION}

\subsection{The radiative components}

The composite SED of AP Librae is shown in Fig. 3. Together with the MOJAVE, SMARTs, Chandra, Swift-UVOT, Swift-XRT, RXTE, Fermi-LAT and HESS data analysed in this work, archival data from NED are reported. In the $30-353 \mathrm{GHz}$ band, the Planck measurements from the Early Release Compact Source Catalogue (ERCSC; Planck Collaboration VII 2011) are in good agreement with the archival data as are the Wide-field Infrared Survey Explorer (WISE; Wright et al. 2010) data in the bands 3.4, 4.6, 12, and $22 \mu \mathrm{m}$.

An extrapolation of the hard X-ray to the optical-UV powerlaw spectrum reported here underestimates the simultaneous UVOT flux by at least 2 orders of magnitude, though the steeply falling UV spectrum possibly connects with the onset of the XRT spectrum. This indicates the presence of an inflection point in the SED widely attributed to a transition from synchrotron to InverseCompton (IC)-dominated radiation. This feature shows that the Compton component of AP Librae is the broadest ever observed in any blazar, spanning more than 10 decades in energy from $\simeq 0.1 \mathrm{keV}$ to $\simeq 1 \mathrm{TeV}$. Indeed, only two other objects of the same class as AP Librae, and hence with broad Compton components, have been detected at VHE energies so far: BL Lac $(z=0.069)$, the first LBL object to be proved as being a VHE emitter (Albert et al. 2007), and S5 0716+714 ( $z=0.310$ ) following an optical trigger (Anderhub et al. 2009). The observed VHE spectrum of the former is not as energetic as AP Librae, and the Xray spectrum of the latter appears to still belong to the synchrotron component. 
Table 3. Parameters of the third-degree polynomial function describing the low and $\mathrm{HE}$ component of AP Librae. The function is of the form $f(E)=p_{0}+$ $p_{1} \log _{10}(E / \mathrm{eV})+p_{2} \log _{10}^{2}(E / \mathrm{eV})+p_{3} \log _{10}^{3}(E / \mathrm{eV})$.

\begin{tabular}{lcccc}
\hline Energy range $(\mathrm{eV})$ & $p_{0}$ & \multicolumn{1}{c}{$p_{1}$} & \multicolumn{1}{c}{$p_{2}$} & \multicolumn{1}{c}{$p_{3}$} \\
\hline $3 \times 10^{-4}$ to 50 & -10.79 & -0.52 & -0.41 & -0.048 \\
50 to $10^{13}$ & -13.36 & 0.82 & -0.068 & -0.001 \\
\hline
\end{tabular}

An empirical characterization of the two radiative components, through a third-degree polynomial fit of each hump in $v F_{v}$ representation (as in e.g. Abdo et al. 2010), is used to estimate the synchrotron and IC peak energies. The values of the parameters obtained from a $\chi^{2}$ fit $^{5}$ are given in Table 3 and the results are represented in the composite SED of Fig. 3. As mentioned above, the SMARTs and the Swift-UVOT measurements in the $V, B$ and $U$ were not used in the fit of the synchrotron peak as well as the data from Falomo et al. (1993). The position of the synchrotron peak is then estimated to be $E_{\mathrm{s} \text {, peak }} \simeq 0.18 \pm 0.06 \mathrm{eV}$, which is compatible with the value of $E_{\mathrm{s} \text {, peak }}=0.26 \mathrm{eV}$ derived by Abdo et al. (2010) on a different data set. The same authors estimated $E_{\text {ic,peak }}=2.6_{-1.4}^{+3.2} \mathrm{GeV}$ for AP Librae in table 13 based on a strong correlation of $E_{\mathrm{ic} \text {,peak }}$ with the HE photon index $\Gamma_{\mathrm{HE}}$, as expressed in their equation (5). ${ }^{6}$ Using the photon index found by Abramowski et al. (2015), which is based on an order of magnitude larger data set, yields a lower but still compatible value of $E_{\text {ic,peak }}=0.9_{-0.5}^{+1.0} \mathrm{GeV}$ and this value was constrained to be below $1 \mathrm{GeV}$ by fitting the HE-VHE data (Abramowski et al. 2015). The polynomial fit presented here yields a much lower value of $E_{\mathrm{ic}, \text { peak }}=17_{-6}^{+24} \mathrm{MeV}$, which can be attributed to use of the entire SED. This is the lowest IC component peak ever measured for a $\mathrm{TeV}$-emitting blazar.

The third-degree polynomial also provides a straightforward estimation of the curvatures $\kappa_{\mathrm{S}}$ and $\kappa_{\mathrm{IC}}$ at the peak positions $E_{\mathrm{s} \text {,peak }}$ and $E_{\text {ic,peak }}$, respectively, which pertain to the widths of each hump. Paggi et al. (2009) show that a relation $\kappa_{\mathrm{s}}=2 \kappa_{\mathrm{IC}}$ is expected in a pure Thomson scattering regime, using a logparabolic parametrization of each of the two humps generated by a single-zone homogeneous SSC model, while $\kappa_{\mathrm{S}}=\kappa_{\mathrm{IC}} / 5$ in the Klein-Nishina $(\mathrm{KN})$ regime. The curvatures found here for AP Librae yield a surprising $\kappa_{\mathrm{s}} \simeq$ $6.6 \kappa_{\text {IC }}$, emphasizing the broadness of the IC component compared to the synchrotron hump, which is hardly possible to reproduce with simple radiative models.

\subsection{Radiative scenarios}

In a one-zone homogenous SSC framework, electrons produce synchrotron photons which are upscattered through the IC mechanism by the same electrons to generate the HE and VHE photons. If this upscattering occurs predominantly in the Thomson regime up to the peak energy, then it becomes possible to constrain the product of the magnetic field $B$ and the Doppler factor $\delta$ for a single-zone homogenous SSC model (following Tavecchio, Maraschi \& Ghisellini 1998, equation 4):

$B \delta=(1+z) \frac{8.6 \times 10^{7} E_{\mathrm{s}, \text { peak }}^{2}}{E_{\mathrm{ic}, \text { peak }}}$,

\footnotetext{
${ }^{5}$ The EBL absorption has been taken into account in the fit.

${ }^{6}$ The quoted uncertainty, not given in their table, is derived from their estimation of an error of 0.7 associated with the estimation of the log of $E_{\text {ic, peak }}$ in equation (5)
}

where the peak energies are expressed in $\mathrm{eV}$. Using the range for $E_{\text {s, peak }}$ found previously and $E_{\mathrm{ic} \text {, peak }}=17 \mathrm{MeV}$ yields $B \delta=0.17 \mathrm{G}$. The value of the break Lorentz factor $\gamma_{\mathrm{b}}$ of the underlying electron distribution can also be derived from the ratio of the peak emission energies as $\sqrt{\frac{3 E_{\text {ic, peak }}}{4 E_{\mathrm{s} \text {, peak }}}} \simeq 8.5 \times 10^{3}$.

Assuming now that the observed synchrotron radiation does not exceed $\simeq 0.1 \mathrm{keV}$ (i.e. the lowest energy bin in the XRT spectrum), which is more likely to belong to the onset of the IC component, then this constrains the maximal Lorentz factor $\gamma_{\max }$ of the underlying electron population through the maximum synchrotron energy

$E_{\mathrm{s}, \max } \simeq \gamma_{\max }^{2} \frac{B \delta m_{\mathrm{e}} c^{2}}{B_{\mathrm{cr}}(1+z)} \leq 0.1 \mathrm{keV}$,

where $B_{\mathrm{cr}}=4.414 \times 10^{13} \mathrm{G}$ is the critical magnetic field leading to

$\gamma_{\max } \leq 10^{5} B^{-1 / 2} \delta^{-1 / 2}$.

Using equations (1) and (2) then yields $\gamma_{\max } \leq 2.4 \times 10^{5}$, which is consistent with being a factor $\sqrt{E_{\mathrm{s}, \max } / E_{\mathrm{s}, \text { peak }}}$ higher than $\gamma_{\mathrm{b}}$ as expected.

Supposing that electrons with an apparent energy of $\delta \gamma_{\max }$ have sufficient energy to upscatter photons to at least the maximal observed Compton energy $E_{\mathrm{ic}, \max } \simeq 1 \mathrm{TeV}$, then the Doppler factor is constrained to a reasonable value of $\delta \geq 10$. If the scattering of $0.1 \mathrm{keV}$ photons occurs in the Thomson regime, the Doppler factor should be such that $4 \gamma_{\max } \times 0.1 \mathrm{keV} \leq \delta m_{\mathrm{e}} c^{2}$. Using the value for $\gamma_{\max }$ found above leads to an unusually high value of $\delta \geq 163$. If however the scattering occurs in the $\mathrm{KN}$ regime for these highest energy seed photons, then $E_{\mathrm{ic}, \max }=\frac{\delta m_{\mathrm{e}} c^{2}}{1+z} \gamma_{\max }$ which, combined with the above constraint (equation 2) on $\gamma_{\max }$, then yields

$B \delta^{-1} \leq 2.3 \times 10^{-3} \mathrm{G}$,

from which follows, using the above constraint $\delta \geq 10$, a reasonable constraint of $B \leq 2.3 \times 10^{-2} \mathrm{G}$. In Appendix A, similar conclusions are drawn for an arbitrary type of seed photons.

Lister et al. (2013) measured an apparent superluminal motion $6.4 \mathrm{c}$. This is compatible with $\delta>10$ for a viewing angle below $<5^{\circ}$ and with $\delta=20$ for 1.7 .

Going further by assuming that photons with energies up to $E_{\text {ic, peak }}$ are produced in the Thomson regime, and the $\simeq 1 \mathrm{TeV}$ photons in the $\mathrm{KN}$ regime, then equations (1) and (3) can be combined to give $B \leq 2 \times 10^{-2} \mathrm{G}$ regardless of the value of $\delta$.

\subsection{Application of an SSC model to the SED}

The time-averaged SED of AP Librae was modelled with a canonical one-zone homogeneous SSC model (Band \& Grindlay 1985). A spherical region of size $R$, with an electron distribution $N_{\mathrm{e}}(\gamma)$, moving with a bulk Doppler factor $\delta$, is filled uniformly with a magnetic field $B$. As in Tavecchio et al. (2010), $N_{\mathrm{e}}(\gamma)$ is described by a broken power law of index $S_{1}$ between $\gamma=1$ and $\gamma_{\mathrm{b}}$ and $S_{2}$ between $\gamma_{\mathrm{b}}$ and $\gamma_{\max }$. The electrons lose their energy by synchrotron emission, producing a field of photons which become the targets for the same electron population through the IC process. The KN effects are taken into account using the Jones kernel (Jones 1968) to compute the IC cross-section.

A tentative model is shown in Fig. 3, where the shape of the electron distribution $\left(S_{1}, S_{2}\right.$ and $\left.\gamma_{\mathrm{b}}\right)$ is constrained by the observed synchrotron component. The remaining parameters $(R, B, \delta$, and the total number of electrons $N_{\mathrm{e}, \text { tot }}$ ) are adjusted to reproduce the onset of the Compton component in the X-rays. The obtained parameters and model curves, as given in Table 4 and Fig. 3, respectively 
Table 4. Parameters of the SSC model presented in this work and from Tavecchio et al. (2010). For both models, $\gamma_{\min }=1$ was used.

\begin{tabular}{lcccccccc}
\hline Model & $\gamma_{\mathrm{b}}$ & $\gamma_{\max }$ & $S_{1}$ & $S_{2}$ & $N_{\mathrm{e}, \text { tot }}$ & $B$ & $R$ & $\delta$ \\
\hline & $10^{4}$ & $10^{4}$ & & & $10^{53}$ & $10^{-2}(\mathrm{G})$ & $10^{16}(\mathrm{~cm})$ & \\
This work & 1.1 & 2.3 & 2 & 4.9 & 5.4 & 0.9 & 3.5 & 20 \\
Tavecchio et al. & 2.0 & 5 & 2 & 4.9 & 0.4 & 1.2 & 1 & 40 \\
\hline
\end{tabular}

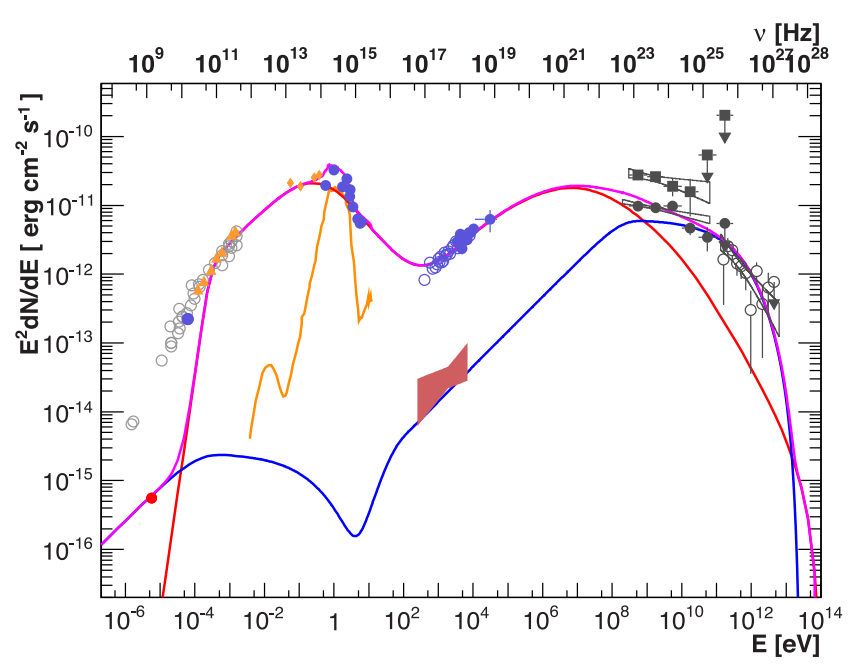

Figure 4. Same as Fig. 3. The red line is the results of the SSC model from the compact component and the blue line is the flux originating from the extended jet; parameters are given in Table 5. Purple line is the sum of both.

[together with the model parameters and curves derived by Tavecchio et al. (2010) for comparison], obey the constraints found in Section 3.2. Not surprisingly, the broad IC component of the SED is difficult to reconcile with the synchrotron distribution using such a simple model, for which strong indications were already presented in Section 3.1.

The SSC calculation reproduces well the lower energy part of the SED, up to the X-rays, but the spectral prediction in the FermiLAT energy range is much softer, as well as about one order of magnitude above the observed HE flux. The direct consequence of the broadness of the IC component is that the HESS flux is largely underestimated. Directly linked to the electron distribution and to the well-measured synchrotron component, this shape can only be affected by the $\mathrm{KN}$ effects, which tend to soften the spectrum, leading inevitably to even larger disagreements.

\subsection{VHE $\gamma$-rays from the extended jet?}

As seen in the previous sections, one-zone SSC models cannot reproduce the broad-band SED of AP Librae. However, Böttcher, Dermer \& Finke (2008) proposed that the Compton-scattering of the cosmic microwave background (CMB) by electrons in an extended kpc-scale jet could make VHE $\gamma$-rays. This model was suggested to explain the hard VHE spectrum from 1ES 1101-232 as observed by HESS (Aharonian et al. 2006, 2007), when EBL attenuation was taken into account with the models available at the time. AP Librae has an extended kpc-scale jet resolved in radio (see Figs 3 and 4) and X-rays (see section 2.2), and it has long been thought that the Compton-scattering of CMB photons could produce the X-rays observed from these extended jets (e.g. Tavecchio et al. 2000; Celotti, Ghisellini \& Chiaberge 2001). Therefore, it seems natural to apply this model to AP Librae to see if the extended jet emission could plausibly make up the VHE $\gamma$-rays. Thus, the broad-band SED of AP Librae has been modelled with a compact, synchrotron/SSC model based on Finke et al. (2008), and an additional component from the extended jet, emitting synchrotron and inverse Comptonscattering of CMB photons (hereafter ICCMB).

The result of this model is shown in Fig. 4, with the model parameters in Table 5. The model parameters are fully described in Finke et al. (2008). The compact component can explain the radio, optical (not including emission that is clearly from the host galaxy), $\mathrm{X}$-ray, and the lower-energy Fermi-LAT $\gamma$-ray data. The extended component can explain the extended radio and X-ray data, as well as the highest $\gamma$-ray emission detected by the LAT and HESS. A double-broken power law was used to describe the electron distribution in the compact component, while only a single broken power law was needed for the electron distribution in the extended component. Parameters in the compact component are broadly comparable to synchrotron/SSC modelling results for other BL Lac objects, including the jet power in electrons being several orders of magnitude greater than that in the magnetic field (e.g. Finke et al. 2008; Abdo et al. 2011a,b,d,c; Aliu et al. 2013, 2014a,b). The extended jet is much closer to equipartition between electron and magnetic field density by design; a model out of equipartition would still be able to reproduce the data. These parameters are also close to previous results for modelling extended jets, although the magnetic field is a bit lower than usual (typically found $>1 \mu \mathrm{G}$; e.g. Tavecchio et al. 2007). This may be because previous ICCMB models of extended jets are for FSRQs rather than BL Lac objects. One hypothesis can be that the magnetic fields in extended jets of BL Lac objects are lower than those in the extended jets of FSRQs.

It should be noted that the ICCMB model for explaining the $\mathrm{X}$-ray emission from extended jets is controversial. It could be that $\mathrm{X}$-rays are instead produced by synchrotron emission from another population of electrons in the extended jet (e.g. Atoyan \& Dermer 2004; Hardcastle 2006). In this alternative framework, HE and VHE emission is unlikely. Recently, Meyer \& Georganopoulos (2014) used Fermi-LAT observations to rule out the ICCMB model for the $\mathrm{X}$-ray emission from the extended jet in the FSRQ 3C 273.

\subsection{Comparison with other LBL objects}

The SEDs of LBL objects detected in VHE $\gamma$-rays challenge singlezone homogeneous SSC radiative models, which usually reproduce reasonably well the time-averaged SEDs of the HBL class.

The most complete simultaneous coverage of the BL Lac was established by Abdo et al. (2011d) during a multi-wavelength campaign including the Fermi-LAT and the X-ray observatories mentioned in this study for the HE part. The X-ray spectrum during that campaign was soft, indicating that its origin was synchrotron radiation rather than Comptonized photons, making for a wider synchrotron $v F_{v}$ distribution than is reported here for AP Librae. The difficulty in this case for modelling BL Lac was that the simulated SED required the energy densities to be far from equipartition. However, a 1997 Beppo-SAX observation (Ravasio et al. 2002) of BL Lac showed a clear IC origin for the $\mathrm{X}$-ray radiation, yielding a narrower synchrotron distribution, for which the SSC model failed to reproduce a reasonable (non-simultaneous) HE spectrum, and an external contribution was added.

The broad Compton distribution of S5 0716+714, with emission up to $\simeq 700 \mathrm{GeV}$, is either an order of magnitude below the best SSC model prediction from Anderhub et al. (2009), or is too wide if the Fermi-LAT spectrum constrains the flux at $E_{\text {ic, peak }}$ (see fig. 6 in Tavecchio et al. 2010; see also the similar situation for BL Lac 
Table 5. Model parameters for the SED shown in Fig. 4. The redshift $z$ is 0.049 .

\begin{tabular}{lccc}
\hline Parameter & Symbol & Compact component & Extended jet \\
\hline Bulk Lorentz factor & $\Gamma$ & 20 & 8 \\
Doppler factor & $\delta_{\mathrm{D}}$ & 20 & 8 \\
Magnetic field (G) & $B$ & 0.05 & $5.6 \times 10^{-7}$ \\
Variability time-scale (s) & $t_{\mathrm{v}}$ & $3.0 \times 10^{4}$ & $1.35 \times 10^{11}$ \\
Comoving radius of blob (cm) & $R_{\mathrm{b}}^{\prime}$ & $1.7 \times 10^{16}$ & $3.08 \times 10^{22}$ \\
First electron spectral index & $p_{1}$ & 2.0 & 2.0 \\
Second electron spectral index & $p_{2}$ & 3.0 & 4.0 \\
Third electron spectral index & $p_{3}$ & 4.2 & 2.0 \\
Minimum electron Lorentz factor & $\gamma_{\text {min }}^{\prime}$ & 1.0 & $4.9 \times 10^{4}$ \\
Break electron Lorentz factor 1 & $\gamma_{\text {brk, }}^{\prime}$ & $2.8 \times 10^{3}$ & \\
Break electron Lorentz factor 2 & $\gamma_{\text {brk,2 }}^{\prime}$ & $6.8 \times 10^{3}$ & $2.0 \times 10^{6}$ \\
Maximum electron Lorentz factor & $\gamma_{\text {max }}^{\prime}$ & $1.0 \times 10^{7}$ & $1.4 \times 10^{44}$ \\
Jet power in magnetic field (erg s ${ }^{-1}$ ) & $P_{\mathrm{j}, B}$ & $2.2 \times 10^{42}$ & $2.8 \times 10^{44}$ \\
Jet power in electrons (erg s ${ }^{-1}$ ) & $P_{\mathrm{j}, \mathrm{e}}$ & $1.7 \times 10^{45}$ & \\
\hline
\end{tabular}

in the same figure). Note that the HE and VHE data were not taken simultaneously in these two LBL objects.

\section{CONCLUSIONS}

Contemporaneous observations of AP Librae with many currently available space- and ground-based instruments have been presented. The data have revealed the broadest Compton distribution of any known blazar to date, which spans from X-ray to $\mathrm{TeV}$ energies.

The SED of AP Librae is difficult to reproduce with a single-zone SSC model: the steep UV spectrum, probably synchrotron emission, does not connect smoothly with the X-ray spectrum, which is underestimated by an order of magnitude if a match is required with the HE $\gamma$-ray spectrum (as was also pointed out by Tavecchio et al. 2010). If a match is required with the X-rays, the Fermi-LAT spectrum is then largely overestimated. The new HESS spectrum further complicates the situation, as none of the previous constraints allows this SSC model to reach the VHE domain, even assuming a predominantly Thomson scattering regime which yields Compton components roughly twice as large in $v F_{v}$ as the synchrotron component. There are ways out of the conundrum but at the cost of increased model complexity. An example is blob-in-jet model, recently proposed by Hervet \& Boisson (2015) to reproduce the SED of Ap Librae. Another possibility is a model where electrons also upscatter soft photons originating outside of the HE emission site. It has been shown in this work that VHE $\gamma$-rays from the extended jet, seen in X-ray, can be produced and can explain the HESS spectrum.

AP Librae is the third of VHE detected LBL-type objects for which single-zone SSC models fail to reproduce the SED, and is currently the only BL Lac type object combining VHE emission and a resolved X-ray jet. The LBL class of VHE emitting objects proves to be an interesting laboratory to test radiative model scenarios, and perhaps to identify parameters on which the LBL-HBL sequence could depend.

\section{ACKNOWLEDGEMENTS}

The Fermi LAT Collaboration acknowledges generous ongoing support from a number of agencies and institutes that have supported both the development and the operation of the LAT as well as scientific data analysis. These include the National Aeronautics and Space Administration and the Department of Energy in the United States, the Commissariat à l'Energie Atomique and the Centre
National de la Recherche Scientifique/Institut National de Physique Nucléaire et de Physique des Particules in France, the Agenzia Spaziale Italiana and the Istituto Nazionale di Fisica Nucleare in Italy, the Ministry of Education, Culture, Sports, Science and Technology (MEXT), High Energy Accelerator Research Organization (KEK) and Japan Aerospace Exploration Agency (JAXA) in Japan, and the K. A. Wallenberg Foundation, the Swedish Research Council and the Swedish National Space Board in Sweden.

Additional support for science analysis during the operations phase is gratefully acknowledged from the Istituto Nazionale di Astrofisica in Italy and the Centre National d'Études Spatiales in France.

This research has made use of data from the MOJAVE data base that is maintained by the MOJAVE team (Lister et al. 2009). The MOJAVE program is supported under NASA-Fermi grant NNX12A087G.

The National Radio Astronomy Observatory is a facility of the National Science Foundation operated under cooperative agreement by Associated Universities, Inc.

This research has made use of the NASA/IPAC Extragalactic Database (NED) which is operated by the Jet Propulsion Laboratory, California Institute of Technology, under contract with the National Aeronautics and Space Administration.

This publication makes use of data products from the WISE, which is a joint project of the University of California, Los Angeles, and the Jet Propulsion Laboratory/California Institute of Technology, funded by the National Aeronautics and Space Administration.

The authors want to acknowledge C.C. Cheung for the VLA radio observation used for contours presented in Fig. 2. AS acknowledges useful discussions with Dan Harris on the problematics of Chandra data analyses. We thank the Swift and RXTE teams for their cooperation in joint observations of AP Librae. This research has made use of data provided by the SIMBAD data base, operated at CDS, Strasbourg, France.

DS was partially supported by the Labex ENIGMASS.

YYK and ABP were supported in part by the Russian Foundation for Basic Research (project 13-02-12103).

TS was partly supported by the Academy of Finland project 274477.

\section{REFERENCES}

Abdo A. A. et al., 2010, ApJ, 716, 30

Abdo A. A. et al., 2011a, ApJ, 736, 131 
Abdo A. A. et al., 2011b, ApJ, 727, 129

Abdo A. A. et al., 2011c, ApJ, 726, 43

Abdo A. A. et al., 2011d, ApJ, 730, 101

Abramowski A. et al., 2015, A\&A, 573, A31

Ackermann M. et al., 2011, ApJ, 743, 171

Aharonian F. et al., 2006, Nature, 440, 1018

Aharonian F. et al., 2007, A\&A, 470, 475

Albert J. et al., 2007, ApJ, 666, L17

Aliu E. et al., 2013, ApJ, 779, 92

Aliu E. et al., 2014a, ApJ, 782, 13

Aliu E. et al., 2014b, ApJ, 797, 89

Anderhub H. et al., 2009, ApJ, 704, L129

Arnaud K. A., 1996, in Jacoby G. H., Barnes J., eds, ASP Conf. Ser. Vol. 101, Astronomical Data Analysis Software and Systems V. Astron. Soc. Pac., San Francisco, p. 17

Atoyan A., Dermer C. D., 2004, ApJ, 613, 151

Band D. L., Grindlay J. E., 1985, ApJ, 298, 128

Bessell M. S., 1990, PASP, 102, 1181

Błażejowski M., Sikora M., Moderski R., Madejski G. M., 2000, ApJ, 545, 107

Bonning E. et al., 2012, ApJ, 756, 13

Böttcher M., Dermer C. D., Finke J. D., 2008, ApJ, 679, L9

Breeveld A. A., Landsman W., Holland S. T., Roming P., Kuin N. P. M., Page M. J., 2011, in McEnery J. E., Racusin J. L., Gehrels N., eds, AIP Conf. Ser. Vol. 1358, GAMMA RAY BURSTS 2010. Am. Inst. Phys., New York, p. 373

Burrows D. N. et al., 2005, Space Sci. Rev., 120, 165

Cardelli J. A., Clayton G. C., Mathis J. S., 1989, ApJ, 345, 245

Celotti A., Ghisellini G., Chiaberge M., 2001, MNRAS, 321, L1

Davis J. E., 2001, ApJ, 562, 575

Falomo R., Bersanelli M., Bouchet P., Tanzi E. G., 1993, AJ, 106, 11

Finke J. D., Dermer C. D., Böttcher M., 2008, ApJ, 686, 181

Franceschini A., Rodighiero G., Vaccari M., 2008, A\&A, 487, 837

Godet O. et al., 2009, A\&A, 494, 775

Hardcastle M. J., 2006, MNRAS, 366, 1465

Harris D. E. et al., 2011, ApJ, 743, 177

Hervet O., Boisson C., 2015, preprint (arXiv:e-prints)

Hofmann W., 2010, Astron. Telegram, 2743, 1

Hyvönen T., Kotilainen J. K., Falomo R., Örndahl E., Pursimo T., 2007, A\&A, 476, 723

Jahoda K., Swank J. H., Giles A. B., Stark M. J., Strohmayer T., Zhang W., Morgan E. H., 1996, in Siegmund O. H., Gummin M. A., eds, SPIE Conf. Ser. Vol 2808. EUV, X-Ray, and Gamma-Ray Instrumentation for Astronomy VII. SPIE, Bellingham, p. 59

Jones F. C., 1968, Phys. Rev., 167, 1159

Jones D. H. et al., 2009, MNRAS, 399, 683

Kalberla P. M. W., Burton W. B., Hartmann D., Arnal E. M., Bajaja E., Morras R., Pöppel W. G. L., 2005, A\&A, 440, 775

Kaufmann S., Wagner S. J., Tibolla O., 2013, ApJ, 776, 68

Lister M. L., Marscher A. P., Gear W. K., 1998, ApJ, 504, 702

Lister M. L. et al., 2009, AJ, 137, 3718

Lister M. L. et al., 2013, AJ, 146, 120

Meyer E. T., Georganopoulos M., 2014, ApJ, 780, L27

Nolan P. L. et al., 2012, ApJS, 199, 31

Padovani P., Giommi P., 1995, ApJ, 444, 567

Paggi A., Massaro F., Vittorini V., Cavaliere A., D'Ammando F., Vagnetti F., Tavani M., 2009, A\&A, 504, 821

Planck Collaboration VII, 2011, A\&A, 536, A7

Poole T. S. et al., 2008, MNRAS, 383, 627

Ravasio M. et al., 2002, A\&A, 383, 763

Roming P. W. A. et al., 2009, ApJ, 690, 163

Sambruna R. M., Gambill J. K., Maraschi L., Tavecchio F., Cerutti R., Cheung C. C., Urry C. M., Chartas G., 2004, ApJ, 608, 698

Sanchez D. A., Fegan S., Giebels B., 2013, A\&A, 554, A75

Schlafly E. F., Finkbeiner D. P., 2011, ApJ, 737, 103

Shaw M. S. et al., 2013, ApJ, 764, 135

Sikora M., Begelman M. C., Rees M. J., 1994, ApJ, 421, 153

Silva L., Granato G. L., Bressan A., Danese L., 1998, ApJ, 509, 103
Tavecchio F., Maraschi L., Ghisellini G., 1998, ApJ, 509, 608

Tavecchio F., Maraschi L., Sambruna R. M., Urry C. M., 2000, ApJ, 544, L23

Tavecchio F., Maraschi L., Wolter A., Cheung C. C., Sambruna R. M., Urry C. M., 2007, ApJ, 662, 900

Tavecchio F., Ghisellini G., Ghirlanda G., Foschini L., Maraschi L., 2010, MNRAS, 401, 1570

The Fermi-LAT Collaboration, 2013, ApJS, 209, 34

Tsujimoto M. et al., 2011, A\&A, 525, A25

Vaughan S., Edelson R., Warwick R. S., Uttley P., 2003, MNRAS, 345, 1271

Wright E. L., 2006, PASP, 118, 1711

Wright E. L. et al., 2010, AJ, 140, 1868

Zensus J. A., Ros E., Kellermann K. I., Cohen M. H., Vermeulen R. C., Kadler M., 2002, AJ, 124, 662

\section{APPENDIX A: CONSTRAINTS FOR AN ARBITRARY FIELD OF SEED PHOTONS}

In leptonic class models, the inverse Compton process is responsible for the HE part of the SED. The seed photons originate either from synchrotron radiation produced within the jet (SSC models) or from a source outside of the jet (external Compton models). In the latter case, the sources can be either the broad-line regions or the dust torus (Sikora, Begelman \& Rees 1994; Błażejowski et al. 2000).

The peak observed energy $E_{\mathrm{s}}$ of an electron with Lorentz factor $\gamma$ is given by

$E_{\mathrm{s}} / m_{\mathrm{e}} c^{2}=\frac{\delta \gamma^{2} B}{(1+z) B_{\mathrm{cr}}}$,

and the Compton-scattered photon energy by

$E_{\mathrm{ic}} / m_{\mathrm{e}} c^{2}=\frac{\delta \gamma^{2} \epsilon_{\mathrm{seed}}^{\prime}}{(1+z)}$

where the energy ${ }^{7}$ of the seed photons is $\epsilon_{\text {seed }}$ (respectively $\epsilon_{\text {seed }}^{\prime}=$ $\delta \epsilon_{\text {seed }}$ in the jet's frame).

Efficient Compton-scattering will occur only for electrons below the KN limit:

$\gamma \leq\left(4 \epsilon_{\text {seed }}^{\prime}\right)^{-1}$.

This KN limit means that Compton-scattered photons will be mainly restricted to energies:

$E_{\text {ic }} / m_{\mathrm{e}} c^{2} \leq \frac{\delta}{16(1+z) \epsilon_{\text {seed }}^{\prime}}$.

The synchrotron photons produced by the electrons having the energy $\left(4 \epsilon_{\text {seed }}^{\prime}\right)^{-1}$ have a peak energy given by:

$E_{\mathrm{s}} / m_{\mathrm{e}} c^{2}=\frac{\delta B}{16(1+z) \epsilon_{\mathrm{seed}}^{\prime 2} B_{\mathrm{cr}}}$.

Combining the last two equations with the constraints on maximal values for $E_{\mathrm{s}} \approx 0.1 \mathrm{keV}$ and $E_{\mathrm{ic}} \approx 1 \mathrm{TeV}$ derived from the observations yields:

$\frac{\delta}{70} \geq \frac{B}{10^{-2} \mathrm{G}}$,

which requires either an unusually high Doppler factor, or an unusually low magnetic field. If the $1 \mathrm{TeV}$ photons are produced by $\mathrm{IC}$ scattering in the $\mathrm{KN}$ regime, equation (A1) becomes

$\gamma \geq\left(4 \epsilon_{\text {seed }}^{\prime}\right)^{-1}$

${ }^{7}$ The notation $E=\epsilon m_{\mathrm{e}} c^{2}$ is adopted here. 
Table B1. Proposed LBL-type objects for VHE observations. The 2FGL name is given in the first column with the position in the second and third columns. The redshift measurement taken from Ackermann et al. (2011) or Shaw et al. (2013) is reported in the fourth column. The name (Column 5) of the counterpart associated with the 2 FGL source was found in the 2LAC catalogue. The last column is the name of the best suited instrument for observations. The sources are ranked by predicted flux above $200 \mathrm{GeV}$.

\begin{tabular}{lllccc}
\hline 2FGL name & \multicolumn{1}{c}{$\alpha_{\mathrm{J} 2000}$} & \multicolumn{1}{c}{$\delta_{\mathrm{J} 2000}$} & Redshift & Association & Instruments \\
\hline 2FGL J1719.3+1744 & $17^{\mathrm{h}} 19^{\mathrm{m}} 13^{\mathrm{s}} .05$ & $17^{\circ} 45^{\prime} 06^{\prime \prime} 4$ & 0.137 & PKS 1717+177 & VERITAS/MAGIC \\
2FGL J0617.6-1716 & $06^{\mathrm{h}} 17^{\mathrm{m}} 33^{\mathrm{s}} .67$ & $-17^{\circ} 15^{\prime} 22^{\prime \prime} .8$ & 0.098 & CRATES J061733.67-171522.8 & HESS \\
2FGL J0738.0+1742 & $07^{\mathrm{h}} 38^{\mathrm{m}} 07^{\mathrm{s}} .39$ & $17^{\circ} 42^{\prime} 19^{\prime \prime} .0$ & 0.424 & PKS 0735+17 & VERITAS/MAGIC - HESS \\
2FGL J1559.0+5627 & $15^{\mathrm{h}} 58^{\mathrm{m}} 48^{\mathrm{s}} .29$ & $56^{\circ} 25^{\prime} 14^{\prime \prime} .1$ & 0.3 & TXS $1557+565$ & VERITAS/MAGIC \\
2FGL J1150.1+2419 & $11^{\mathrm{h}} 50^{\mathrm{m}} 19^{\mathrm{s}} .21$ & $24^{\circ} 17^{\prime} 53^{\prime \prime} 8$ & 0.2 & B2 $1147+24$ & VERITAS/MAGIC \\
2FGL J0712.9+5032 & $07^{\mathrm{h}} 12^{\mathrm{m}} 43^{\mathrm{s}} .68$ & $50^{\circ} 33^{\prime} 22^{\prime \prime} .7$ & 0.502 & GB6 J0712+5033 & VERITAS/MAGIC \\
\hline
\end{tabular}

and the observed photon energy is (Tavecchio et al. 1998)

$E_{\mathrm{c}} / m_{\mathrm{e}} c^{2}=\frac{\delta \gamma}{(1+z)}$.

Then equation (A2) reads

$$
\frac{\delta}{17.5} \leq \frac{B}{10^{-2} \mathrm{G}}
$$

which is a reasonable constraint. Note that this calculation applies no matter what the seed photon source is (broad-line region or dust torus or synchrotron photons produced within the jet), illustrating the difficulties of either radiative scenarios to account for the main SED features of AP Librae in the Thomson regime.

\section{APPENDIX B: CANDIDATES FOR VHE OBSERVATIONS}

The detection of AP Librae by the HESS telescopes has revealed the broadest IC component for a blazar with a peak position at very low energy. Unfortunately, only a handful of LBL-type objects have yet been detected at VHEs. To decide if AP Librae is a special case or a typical representative of the LBL class, other LBL objects have to be observed by Čerenkov telescope and detected at VHE.

Due to their limited field of view $\left(\approx 5^{\circ}\right)$, an extragalactic survey performed by Čerenkov telescopes is not possible yet. As a consequence, good targets for observations have to be found based on multi-wavelengths data. In this Appendix, six LBL-type objects, present in the second catalogue of Fermi sources (2FGL; Nolan et al. 2012), were selected based on their possible VHE emission. The 2FGL best-fitting power law, measured in the 100 MeV-100 GeV band, was extrapolated above $200 \mathrm{GeV}$ and EBL correction was made based on the Franceschini, Rodighiero \& Vaccari (2008) model. The redshift information was extracted either from the second catalogue of AGN (2LAC; Ackermann et al. 2011) or from Shaw et al. (2013). Sources without redshift measurement were excluded and only sources classified as a BL Lac of the LBL class were retained. Note that AP Librae appeared to be the first on this list when building it.

The names of six candidates, ranked by predicted flux above $200 \mathrm{GeV}$, are given in Table B1. For illustration, their SEDs, built from archival data using the ASDC SED builder, ${ }^{8}$ are presented in Fig. B1. Two out of the six sources can be observed by HESS and five by the northern facilities (VERITAS and MAGIC). Despite its location and with a redshift of $z=0.424$, the source 2FGL J0738.0+1742 can be well suited for HESS II telescope observations given the lower energy threshold $(50 \mathrm{GeV})$ of the instrument. The redshifts of 2FGL J1150.1+2419 and 2FGL J1150.1+2419, found in the 2LAC, were not confirmed by Shaw et al. (2013). Five out of six are also present in the first Fermi-LAT Catalogue of Sources Above $10 \mathrm{GeV}$ (1FHL; The Fermi-LAT Collaboration 2013, see Fig. B1).

\footnotetext{
${ }^{8}$ http://tools.asdc.asi.it/SED/
} 

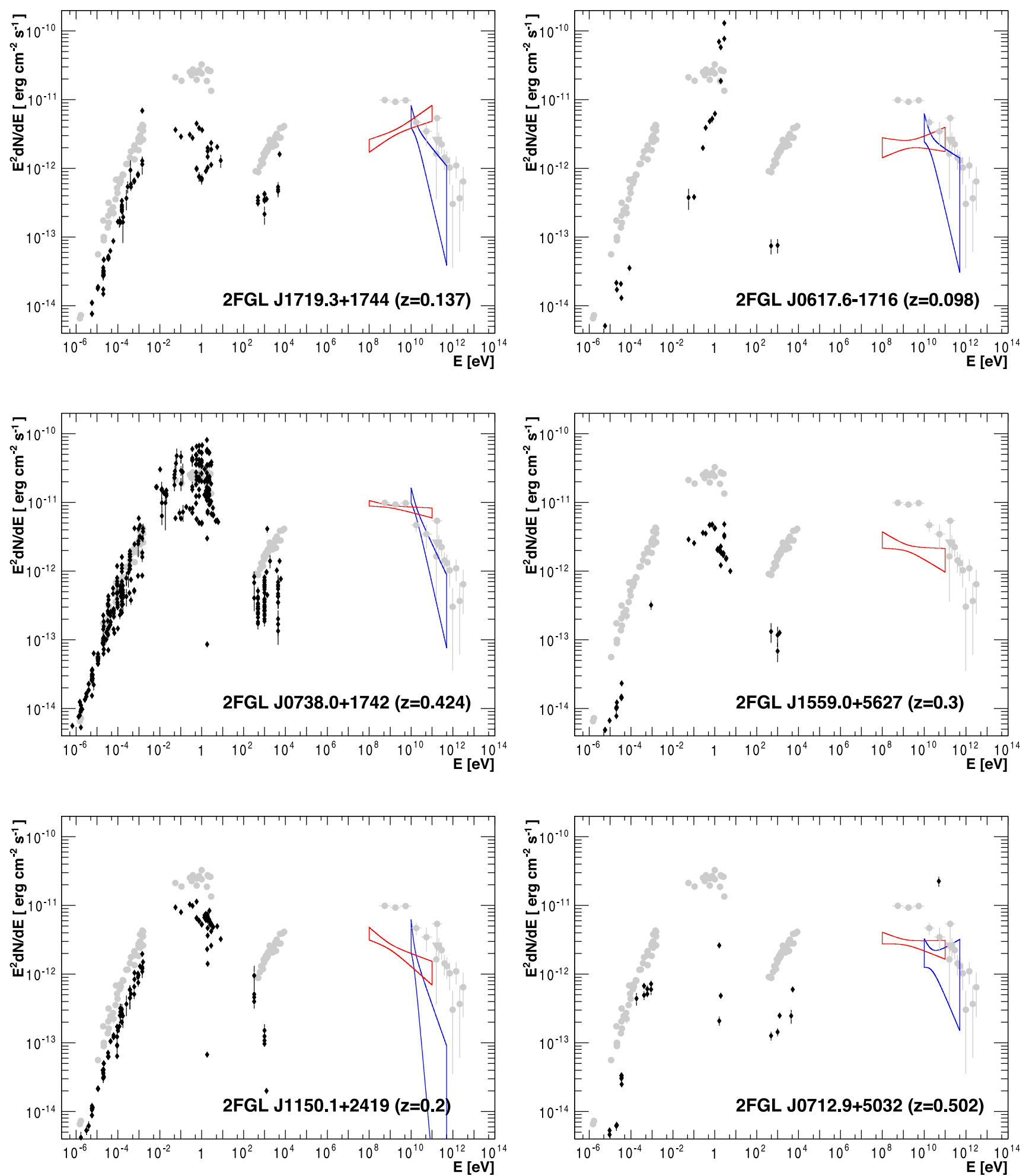

Figure B1. SEDs for the six LBL objects selected. The black points are archival data while the respective red and blue butterflies are the 2FGL and 1FHL measurements. Grey points are the AP Librae data presented in this work.

This paper has been typeset from a $\mathrm{T}_{\mathrm{E}} \mathrm{X} / \mathrm{L} \mathrm{T} \mathrm{E}_{\mathrm{E}} \mathrm{X}$ file prepared by the author. 